\title{
Choline PET based dose-painting in prostate cancer - Modelling of dose effects
}

Maximilian Niyazi ${ }^{1}$, Peter Bartenstein ${ }^{2}$, Claus Belka ${ }^{1}$, Ute Ganswindt ${ }^{1 *}$

\begin{abstract}
Background: Several randomized trials have documented the value of radiation dose escalation in patients with prostate cancer, especially in patients with intermediate risk profile. Up to now dose escalation is usually applied to the whole prostate. IMRT and related techniques currently allow for dose escalation in sub-volumes of the organ. However, the sensitivity of the imaging modality and the fact that small islands of cancer are often dispersed within the whole organ may limit these approaches with regard to a clear clinical benefit. In order to assess potential effects of a dose escalation in certain sub-volumes based on choline PET imaging a mathematical doseresponse model was developed.
\end{abstract}

Methods: Based on different assumptions for $\alpha / \beta, \gamma 50$, sensitivity and specificity of choline PET, the influence of the whole prostate and simultaneous integrated boost (SIB) dose on tumor control probability (TCP) was calculated. Based on the given heterogeneity of all potential variables certain representative permutations of the parameters were chosen and, subsequently, the influence on TCP was assessed.

Results: Using schedules with 74 Gy within the whole prostate and a SIB dose of 90 Gy the TCP increase ranged from 23.1\% (high detection rate of choline PET, low whole prostate dose, high $\gamma 50 /$ ASTRO definition for tumor control) to $1.4 \%$ TCP gain (low sensitivity of PET, high whole prostate dose, CN +2 definition for tumor control) or even $0 \%$ in selected cases. The corresponding initial TCP values without integrated boost ranged from $67.3 \%$ to $100 \%$. According to a large data set of intermediate-risk prostate cancer patients the resulting TCP gains ranged from $22.2 \%$ to $10.1 \%$ (ASTRO definition) or from $13.2 \%$ to $6.0 \%$ (CN +2 definition).

Discussion: Although a simplified mathematical model was employed, the presented model allows for an estimation in how far given schedules are relevant for clinical practice. However, the benefit of a SIB based on choline PET seems less than intuitively expected. Only under the assumption of high detection rates and low initial TCP values the TCP gain has been shown to be relevant.

Conclusions: Based on the employed assumptions, specific dose escalation to choline PET positive areas within the prostate may increase the local control rates. Due to the lack of exact PET sensitivity and prostate $\alpha / \beta$ parameter, no firm conclusions can be made. Small variations may completely abrogate the clinical benefit of a SIB based on choline PET imaging.

\section{Introduction}

Several randomized trials have documented a clear doseresponse relationship for prostate cancer. Although not employing modern IMRT techniques the M. D. Anderson phase III dose escalation trial was the first randomized trial to prove 78 Gy vs. 70 Gy. It resulted in better biochemical control for the higher radiation dose

\footnotetext{
* Correspondence: ute.ganswindt@med.uni-muenchen.de ${ }^{1}$ Department of Radiation Oncology, Ludwig-Maximilians-University München, Marchioninistr. 15, 81377 München, Germany
}

in patients with intermediate-risk features [1]. Other groups obtained similar results [2-6]. This interpretation is corroborated by population based approaches showing that only doses $\geq 72$ Gy are associated with adequate tumor control $[7,8]$.

The implementation of IMRT into clinical practice of prostate cancer radiation treatment enables the physician to increase the doses in focal areas of the gland, which is in contrast to the central dogma in radiation oncology to strive for a homogeneous dose to the target volume [9]. However, this approach might have two 
advantages: Firstly the dose escalation is limited to a minor part of the target volume and thus, the probability of side effects should be lowered [10]. Secondly the biological efficacy may be increased by the use of higher doses per fraction.

The first who addressed this issue were Pickett, Xia and colleagues [11,12], later on further studies were conducted [13,14], also in case of high-risk prostate cancer [15]. Li et al. reported a new IMRT simultaneous integrated boost (SIB) strategy that irradiates prostate via hypo-fractionation while irradiating pelvic nodes with the conventional fractionation. Compared to the conventional two-phase treatment, the proposed SIB technique offers potential advantages, including better sparing of critical structures leading to less incontinence, rectal bleeding, irritative symptoms [16-20] or urethral toxicity [21], more efficient delivery, shorter treatment duration, and better biological efficacy [22]. Fonteyne et al. reported that addition of an IMRT SIB to an intra-prostatic lesion (defined by magnetic resonance imaging) did not increase the severity or incidence of acute toxicity [23]. Furthermore new techniques like volumetric modulated arcs, helical tomotherapy or IMPT additionally showed improvements in conformal avoidance relative to fixed beam IMRT $[24,25]$.

Despite the technical advances in radiotherapy the optimal treatment for prostate cancer strongly depends on the accuracy of tumor characterization and staging. Positron emission tomography (PET) is an exquisitely sensitive molecular imaging technique using positronemitting radioisotopes coupled to specific ligands [26].

Different PET tracers, including $\left[{ }^{11} \mathrm{C}\right]$ choline, $\left[{ }^{18} \mathrm{~F}\right]$ choline and $\left[{ }^{11} \mathrm{C}\right]$ acetate, have been described for the detection of prostate cancer. However, larger trials are still needed to establish their final clinical value concerning the primary detection and the staging of prostate cancer [27].

In principle, signal-generation is based on an increased choline metabolism in prostate cancer leading to an increased up-take in tumor tissue compared to that of benign tissue [28]. However, benign prostate hyperplasia and inflammatory changes may also lead to increased uptake thereby lowering the specificity of the PET signal.

A precise volumetric assessment of PET signals is of rising importance for radiotherapy (RT) planning [29]. The use of choline PET/CT data to detect tumor spots within the prostate has been analyzed and first clinical experiences in lymph node-positive patients were reported [30]. In this regard, Ciernik et al. investigated the utility of F-18-choline PET signals to serve as a target for semi-automatic segmentation for forward treatment planning of prostate cancer. F-18-choline PET and $\mathrm{CT}$ scans of ten patients with histologically proven prostate cancer without extra-capsular tumor extension were acquired using a combined PET/CT scanner. Planning target volumes (PTV's) derived from CT and F-18choline PET yielded comparable results. 3D-conformal planning with CT or F-18-choline PET resulted in comparable doses to the rectal wall. Choline PET signals of the prostate provided adequate spatial information to be used for standardized PET-based target volume definition [31].

As PET allows for detection of small lesions within the prostate and modern IMRT techniques can be used for integrated focal boosting, it is evident to use PET information in order to escalate the dose within defined tumor spots also called biologically guided radiotherapy [32]. This type of selective dose-escalation has already been implemented successfully using spectroscopic MRI data $[23,33,34]$. Although doing so may be intuitively reasonable, the true effect of such procedures is strongly influenced by a multitude of factors. We therefore attempted to develop a method to estimate the increase of local tumor control using an IMRT SIB to choline PET positive hotspots within the gland. The computations were done in a putative intermediate-risk collective reflecting the fact that these patients will have the most benefit by any dose escalation approach.

\section{Methods}

The best currently available dataset for dose-response relationships in prostate cancer was derived from a study of 235 low-risk and 382 intermediate-risk patients treated between 1987 and 1998 with external beam RT alone at the M. D. Anderson Cancer Center [35].

Local control (biochemical no evidence of disease) was defined in two different ways; Firstly, ASTRO definition was employed: Time to PSA failure is defined as the end of RT to the mid-point between the PSA nadir and the first PSA rise [35]. Secondly, the Houston definition defines biochemical failure as PSA rise of $\geq 2 \mathrm{ng} / \mathrm{ml}$ above the current nadir PSA $(\mathrm{CN}+2)$ [36-38]. In both settings detectable local, nodal and distant relapses as well as initiation of hormonal treatment are scored as failures.

In order to develop a mathematical TCP model for prostate cancer, we firstly assumed the prostate to be a geometrical structure subdivided into a fixed number of voxels (defining their volume as $v_{i}=1$ ). Voxels including tumor cells are called tumorlets.

$\mathrm{N}$ is defined as the number of clonogenic cells within the tumor, $V$ as the volume of the target volume and $n_{i}$ is defined as the density of tumor cells within a tumorlet. We furthermore assumed that all tumorlets have the same density of clonogenic cells. In order to achieve this in practice one has to define the voxels as sufficiently small. 
The tumor control probability (TCP) is modelled as a Poisson distribution [39]. In such a geometrical setting it is defined as:

$$
T C P=\prod_{i} e^{-n_{i} S F_{i}}
$$

$\mathrm{SF}_{\mathrm{i}}$ is the surviving fraction within the single subvolume with the running index $\mathrm{i}$ (ranging from 1 to $\mathrm{m}$ $\left.=\mathrm{V} / \mathrm{v}_{\mathrm{i}}\right)$. Using the well-known linear-quadratic model the surviving fraction can be calculated as:

$$
S F=e^{-\alpha n d_{j}}\left(1+\frac{d_{j}}{\alpha / \beta}\right)
$$

with $\mathrm{d}_{j}$ as single dose (usually 1.8 or $2 \mathrm{~Gy}$ ), $\mathrm{n}$ as the number of fractions and $\alpha, \beta$ as the parameters from the linear-quadratic model which refer to the radio-sensitivity of the tumor cells ( $\alpha$ represents lethal lesions made by one-track action and $\beta$ accounts for lethal lesions made by two-track action, [40]). In this formula the tumor doubling time is not considered.

Relevant $\alpha / \beta$ ratios can be obtained from both in vitro experiments and clinical fractionation studies and give the dose where linear and quadratic effect are equal according to total cell kill [41] whereas in vitro data do not necessarily predict the radio-sensitivity of tissues in clinical radiotherapy. There is a wide variation of $\alpha / \beta$ values for prostate cancer in the literature with the exact value of $\alpha / \beta$ being still unknown [41-51].

Thus, the following calculations were based on the values determined by Fowler et al. $(\alpha / \beta=1.5 \mathrm{~Gy}, \alpha=$ $\left.0.04 \mathrm{~Gy}^{-1}\right)$ [43], Wang et al. $(\alpha / \beta=3.1 \mathrm{~Gy}, \alpha=0.15$ $\left.\mathrm{Gy}^{-1}[49,52]\right)$ and Valdagni et al. $(\alpha / \beta=8.3$ Gy $[46,48])$.

Another relevant parameter to describe the TCP is the slope of the killing curve $(\gamma 50)$ which relates to the number of clonogens within the tumor in the following way [53]:

$$
\gamma 50=\frac{\ln 2}{2 \ln \left(\frac{N}{\ln 2}\right)}
$$

Cheung et al. calculated a $\gamma 50$ value of 2.2 [1.1-3.2, 95\% CI] and TCD50 = 67.5 Gy [65.5-69.5 Gy, 95\% CI] (ASTRO definition) or $\gamma 50=1.4[0.2-2.5,95 \% \mathrm{CI}]$ and TCD50 $=57.8$ Gy [49.8-65.9 Gy, 95\% CI $](\mathrm{CN}+2$ definition) for intermediate-risk patients [35]. The corresponding TCP curves are shown in Figure 1.

Those voxels not containing a clonogenic cell (pure prostate tissue) do not contribute to the overall TCP as the corresponding factor equals 1.

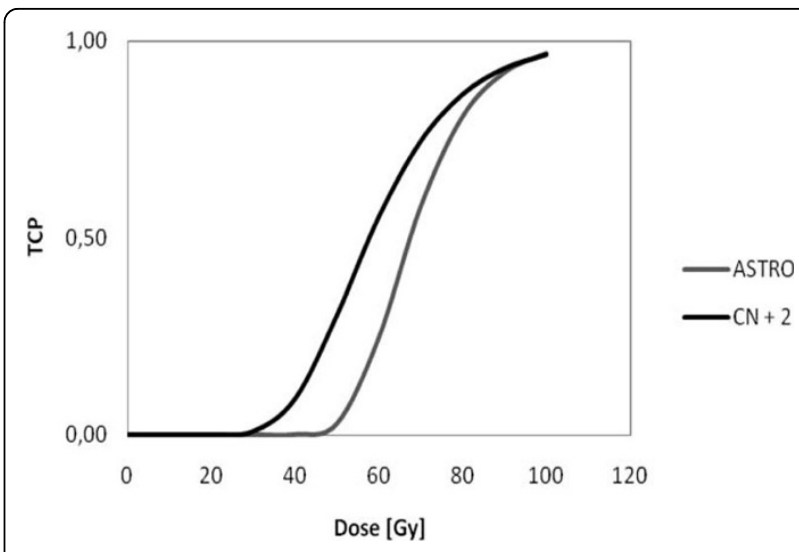

Figure 1 Tumor control probability curves for both definitions of local control derived by data of Cheung et al. (RT of the whole prostate).

Summarizing all these equations, and after some algebraic manipulations keeping in mind that $\mathrm{v}_{\mathrm{i}}=1$, one obtains:

$$
T C P_{\text {SIB }} / T C P_{\text {conv }}=e^{-\Delta S F \times N}
$$

$\Delta \mathrm{SF}$ denotes the difference between boosted and conventional surviving fraction (conventional means without boost, but 3D-conformal RT or IMRT technique). This expression has to be corrected due to the limited sensitivity in detecting all clonogenic cells. The sensitivity values for choline PET range from $81 \%$ (for a SUV of 2.65) [54] down to 73\% [28,55] or 64\% [56] (Additional file 1 offers the possibility to specify different parameters for intermediate-risk prostate cancer to calculate the effect of an IMRT SIB).

This is a simplified picture of reality as the sensitivity of detecting tumor cells within the prostate is dependent on the size or more precise intensity of the enhancing tumor lesion. Partial volume effects can severely affect images both qualitatively and quantitatively: For any hot lesion of a small size and embedded in a colder background, this effect spreads out the signal. It typically occurs whenever the tumor size is less than 3 times the full width at half maximum (FWHM) of the reconstructed image resolution. The maximum value in the hot tumor then will be lower than the actual maximum value. A small tumor will look larger but less aggressive than it actually is [57]. The model assumes the detection rate for the sake of simplicity size-independent and constant, the aforementioned sensitivities from the literature are taken as best guesses for the detection rate.

The model used for our calculation is based on a number of additional assumptions. Thus, several 
shortcomings have to be taken into account when interpreting the data:

1) The assumption of a homogeneous density of clonogenic tumor-cells is not obvious. There may be islands within the prostate with a higher clonogenic density. However, this is no strict contradiction to our assumption as the sub-voxels may be scaled down until only empty voxels and voxels with a small but uniform number of clonogenic cells remain left.

2) The given model is incapable of reflecting biological sub-volume effects adequately: For example, one may assume that hypoxic areas within high-density tumor foci may cause a locally enhanced radio-resistance. Since all values used for our calculation are based on whole organ TCPs, the given model ignores issues of focally increased resistance.

3) Biologically, a complex feedback between the tumor and surrounding normal tissue exists. For example, the release of certain cytokines after radiation damage may influence the surrounding tumor tissue and vice versa. Again the given model is not able to integrate the putative interaction of adjacent clonogenic tumor and stroma cells.

4) It is assumed that all clonogenic cells within the tumor have a uniform radiosensitivity.

All these effects may be in place but do not seem to have much influence in practice. One prominent example is the comparison between primary and salvage radiotherapy.

After prostatectomy with positive surgical margins adjuvant radiotherapy improves disease-free survival rates and thus it is discussed as a new standard of adjuvant treatment in selected cases [58]; in cases of local relapse, salvage radiotherapy is the only potentially curative treatment approach [59]. The doses being necessary to control microscopic tumor seem to be higher than initially expected and to be similar to those for macroscopic tumor within the setting of a primary treatment [60].

\section{Results}

The relevant parameters fed into our model in order to calculate the increase in whole organ TCP are: Sensitivity of choline PET, $\alpha, \alpha / \beta, \gamma 50$, whole prostate dose, SIB dose and dose per fraction.

In order to present the calculations different representative scenarios have been tested:

\section{High sensitivity of choline PET, low whole prostate dose, high $\gamma 50$ (ASTRO consensus), Fowler's $\alpha / \beta$}

This parameter set was chosen to calculate a putative maximum TCP increase: Choline PET sensitivity was set to $81 \%$ and 74 Gy were chosen as homogeneous prostate dose. $\alpha / \beta$ was set to $1.5 \mathrm{~Gy}\left(\alpha=0.04 \mathrm{~Gy}^{-1}\right), \gamma 50$ was chosen according to Cheung's data with the ASTRO definition. As shown in Figure 1 this parameter set leads to a higher steepness of the TCP curve. The results are shown in Table 1. The TCP in this setting with homogeneous dose of 74 Gy within the prostate was $67.3 \%$ and was improved by $23.1 \%$ up to $90.4 \%$ using a SIB.

\section{High sensitivity of choline PET, low whole prostate dose, low $\gamma 50$ ( $\mathrm{CN}+2$ definition), Fowler's $\alpha / \beta$}

In contrast, one may assume a parameter set with slightly less optimal conditions for a SIB. Table 2 summarizes the results when assuming a higher detection rate for PET (81\%), a low homogeneous whole prostate dose (74 Gy), a SIB dose of 90 Gy and radio-sensitivity parameters as described by Fowler et al. $(\alpha / \beta=1.5 \mathrm{~Gy}$, $\alpha=0.04 \mathrm{~Gy}^{-1}$ ) and $\gamma 50$ taken again from Cheung's data but this time according to the $\mathrm{CN}+2$ definition. The calculated TCP without SIB was $96.0 \%$ which leaves only an increase of $2.9 \%$ with a SIB.

This result is basically driven by a high initial control probability. In reality the initial clinical control probability is lower [35].

Table 1 TCP-increase for high sensitivity of choline PET, low whole prostate dose, high $\gamma 50$ (ASTRO consensus) and Fowler's $\alpha / \beta$

\begin{tabular}{lcccccccc}
\hline $\boldsymbol{\alpha}\left[\mathrm{Gy}^{-1}\right]$ & $\boldsymbol{\alpha} / \boldsymbol{\beta}$ [Gy] & $\boldsymbol{\gamma 5 0}$ & Det. rate PET [\%] & Dose [Gy] & SIB [Gy] & Single dose [Gy] & TCP $_{\text {conv }}[\%]$ & TCP Increase [\%] \\
\hline 0.04 & 1.5 & 2.2 & 81 & 74 & 90 & 2 & 67.3 & 23.1 \\
\hline
\end{tabular}

Calculation of the increase in TCP with whole prostate dose of 74 Gy after boosting choline PET positive regions within the prostate up to 90 Gy. $\alpha$ and $\alpha / \beta$ are estimated from Fowler's data and $\gamma 50$ from Cheung's data (ASTRO definition). For choline PET a high sensitivity was used.

Table 2 TCP-increase for high sensitivity of choline PET, low whole prostate dose, low $\gamma 50(\mathrm{CN}+2$ definition) and Fowler's $\alpha / \beta$

\begin{tabular}{lcccccccc}
\hline $\boldsymbol{\alpha}$ [Gy $\left.^{-1}\right]$ & $\boldsymbol{\alpha} / \boldsymbol{\beta}$ [Gy] & $\boldsymbol{\gamma} \mathbf{5 0}$ & Det. rate PET [\%] & Dose [Gy] & SIB [Gy] & Single dose [Gy] & TCP $_{\text {conv }}[\%]$ & TCP Increase [\%] \\
\hline 0.04 & 1.5 & 1.4 & 81 & 74 & 90 & 2 & 96.0 & 2.9
\end{tabular}

Calculation of the TCP-increase after boosting choline PET positive regions within the prostate up to $90 \mathrm{~Gy}$. $\alpha / \beta$ is estimated from Fowler's data and $\gamma 50$ from Cheung's data (CN +2 definition). For choline PET a high sensitivity was used. 
3. Low sensitivity of choline PET, high whole prostate dose, low $\gamma 50(\mathrm{CN}+2$ definition), Fowler's $\alpha / \beta$

A "worst case" scenario is considered where a low sensitivity of PET is presumed, the homogeneous whole prostate dose is high (see Table 3, 78 Gy along the dose concept of the M. D. Anderson trial [1]), $\alpha / \beta$ is low and $\gamma 50$ is less steep than the corresponding ASTRO value.

Based on these assumptions the gain of a SIB is low, as the initial TCP is again very high (97.0\%) and as the remaining SIB effect is small (1.4\%). Again, this result is in contrast to clinical reality reflected in the Cheung data [35].

\section{High sensitivity of choline PET, low whole prostate} dose, $\gamma 50$ arbitrary, Wang's $\alpha / \beta$

Using $\alpha / \beta$ and $\alpha$ values originally obtained by Wang et al. one obtains independently of $\gamma 50$ or the whole organ dose a TCP of $100 \%$ which leaves no benefit for a SIB (Table 4). This result is probably due to the fact that the respective $\gamma 50$ as well as $\alpha / \beta$ parameters were derived from independent clinical trials.
5. Different sensitivities of choline PET, low whole prostate dose, different $\alpha / \beta$ values, calculated $\alpha$, high $\gamma 50$ (ASTRO definition)

In order to circumvent the problem of overestimating the initial TCP one can try to reproduce the $M$. D. Anderson data (Cheung et al.) employing different $\alpha / \beta$ values (Fowler, Wang, Valdagni) and fitting an optimal $\alpha$ value to finally achieve a realistic concordance between observed TCD50 and calculated TCD50 value.

In Table 5 the ASTRO consensus was used for the definition of tumor control, leading to a steeper TCP curve (see Figure 1). Using a low whole prostate dose (74 Gy), the baseline tumor control was $68.7 \%$. In this setting the SIB mediated TCP increase was strongly dependent on the sensitivity of the choline PET. Assuming a sensitivity rate of $81 \%$, the TCP was increased by $22.2 \%$, for $64 \%$ the increase was lowered to $17.0 \%$.

Using higher $\alpha / \beta$ values automatically resulted in a lower TCP gain. This difference is based on the fact that in the given model $\alpha$ was optimized with fixed $\gamma 50$ and $\alpha / \beta$, resulting in different TCP curves.

Table 3 TCP-increase for low sensitivity of choline PET, high whole prostate dose, low $\gamma 50(\mathrm{CN}+2$ definition) and Fowler's $\alpha / \beta$

\begin{tabular}{lcccccccc}
\hline $\boldsymbol{\alpha}\left[\mathrm{Gy}^{-1}\right]$ & $\boldsymbol{\alpha} / \boldsymbol{\beta}[\mathrm{Gy}]$ & $\boldsymbol{\gamma} \mathbf{5 0}$ & Det. rate PET [\%] & Dose [Gy] & SIB [Gy] & Single dose [Gy] & TCP $_{\text {conv }}[\%]$ & TCP Increase [\%] \\
\hline 0.04 & 1.5 & 1.4 & 64 & 78 & 90 & 2 & 97.0 & 1.4
\end{tabular}

Calculation of the TCP-increase after boosting choline PET positive regions within the prostate up to 90 Gy; the whole organ dose was set to $78 \mathrm{~Gy}$. $\alpha / \beta$ is estimated from Fowler's data and $\gamma 50$ from Cheung's data ( $C N+2$ definition). For choline PET a low sensitivity was used.

Table 4 TCP-increase for high sensitivity of choline PET, low whole prostate dose, $\gamma 50$ arbitrary and Wang's $\alpha / \beta$

\begin{tabular}{lcccccccc}
\hline $\boldsymbol{\alpha}\left[\mathrm{Gy}^{-1}\right.$ ] & $\boldsymbol{\alpha} / \boldsymbol{\beta}$ [Gy] & $\boldsymbol{\gamma 5 0}$ & Det. rate PET [\%] & Dose [Gy] & SIB [Gy] & Single dose [Gy] & TCP $_{\text {conv }}$ [\%] & TCP Increase [\%] \\
\hline 0.15 & 3.1 & 1.4 & 81 & 74 & 90 & 2 & 100 & 0 \\
0.15 & 3.1 & 2.2 & 81 & 74 & 90 & 2 & 100 & 0 \\
\hline
\end{tabular}

Calculation of the TCP-increase after boosting choline PET positive regions within the prostate up to 90 Gy. $\alpha / \beta$ is estimated from Wang's data and $\gamma 50$ arbitrary. For choline PET a high sensitivity was assumed.

Table 5 TCP-increase for different sensitivities of choline PET, low whole prostate dose, different $\alpha / \beta$ values, calculated $\alpha$ and high $\gamma 50$ (ASTRO definition)

\begin{tabular}{|c|c|c|c|c|c|c|c|c|}
\hline$\alpha\left[\mathrm{Gy}^{-1}\right]$ & $\alpha / \beta[G y]$ & $\gamma 50$ & Det. rate PET [\%] & Dose [Gy] & SIB [Gy] & Single dose [Gy] & $\mathrm{TCP}_{\text {conv }}[\%]$ & TCP Increase [\%] \\
\hline 0.04 & 1.5 & 2.2 & 81 & 74 & 90 & 2 & 68.7 & 22.2 \\
\hline 0.04 & 1.5 & 2.2 & 64 & 74 & 90 & 2 & 68.7 & 17.0 \\
\hline 0.06 & 3.1 & 2.2 & 81 & 74 & 90 & 2 & 68.7 & 21.4 \\
\hline 0.06 & 3.1 & 2.2 & 64 & 74 & 90 & 2 & 68.7 & 16.4 \\
\hline 0.08 & 8.3 & 2.2 & 81 & 74 & 90 & 2 & 68.7 & 20.1 \\
\hline 0.08 & 8.3 & 2.2 & 64 & 74 & 90 & 2 & 68.7 & 15.4 \\
\hline
\end{tabular}

Calculation of the TCP-increase after boosting choline PET positive regions within the prostate up to $90 \mathrm{~Gy}$. $\alpha / \beta$ was set to either Fowler's/Wang's or Valdagni's value, $\alpha$ was analytically determined in order to achieve agreement between calculated TCD50 and TCD50 obtained by Cheung et al. $\gamma 50$ was again taken from Cheung's data (ASTRO definition). 
6. Different sensitivities of choline PET, high whole prostate dose, different $\alpha / \beta$ values, calculated $\alpha$, high $\gamma 50$ (ASTRO definition)

Compared to Table 5 in Table 6 a higher whole prostate dose $(78$ Gy) was used. The initial TCP could be improved to $77.2 \%$. The increase in TCP by the given SIB dose was lower ranging from 14.9\% (high PET sensitivity, low $\alpha / \beta$ ) to $10.1 \%$ (low detection rate, high $\alpha / \beta$ ).

\section{Different sensitivities of PET, low whole prostate dose,} different $\alpha / \beta$ values, calculated $\alpha$, low $\gamma 50$ (CN +2 definition)

In Table 7 the $\mathrm{CN}+2$ consensus was used to define tumor control, leading to less steep TCP curves (see Figure 1). Again, a low whole prostate dose was used; the baseline tumor control then was calculated to be $80.0 \%$. Similarly to the previous scenario, the TCP-increase by a given SIB was also strongly related to the assumed sensitivity of the choline PET. Using a sensitivity of $81 \%$ the TCP was increased by $13.2 \%$ compared to $22.2 \%$ in the same setting employing the ASTRO definition. In contrast, for $64 \%$ sensitivity the increase was only $10.3 \%$. Replacing the given $\alpha / \beta$ by higher values resulted in lower TCP gains. The lowest increase for TCP was seen for Valdagni's $\alpha / \beta$ with a low choline PET sensitivity: $9.0 \%$.
8. Different sensitivities of PET, high whole prostate dose, different $\alpha / \beta$ values, calculated $\alpha$, low $\gamma 50(\mathrm{CN}+2$ definition)

Compared to Table 7 in Table 8 a higher whole prostate dose was used (78 Gy). The initial TCP could be improved to $80 \%$. The increase in TCP was lower as it ranged from $9.1 \%$ (high detection rate of PET, low $\alpha / \beta$ ) to $6.0 \%$ (low detection rate, high $\alpha / \beta$ ).

\section{Discussion}

Using a simplified mathematical model allowed us to determine the increase in TCP after an IMRT SIB based on choline PET positive intra-prostatic lesions. The model has been based on several fundamental assumptions including uniform clonogenic cell density, no interaction between adjacent tumor cells, no sub-volume effects and a uniform radio-sensitivity of all tumor cells. Furthermore the model does not consider population differences or time factors [61]. This model is substantiated by the fact that doses being needed to control microscopic tumor in an adjuvant/salvage setting seem to be almost as high as those used in primary therapy for macroscopic tumors [60].

It was shown that a SIB mediated increase of the given TCP is strongly dependent on the sensitivity of the choline PET, the $\gamma 50$-value with special emphasis on

Table 6 TCP-increase for different sensitivities of choline PET, high whole prostate dose, different $\alpha / \beta$ values, calculated $\alpha$ and high $\gamma 50$ (ASTRO definition)

\begin{tabular}{|c|c|c|c|c|c|c|c|c|}
\hline$\alpha\left[\mathrm{Gy}^{-1}\right]$ & $\alpha / \beta$ [Gy] & $\gamma 50$ & Det. rate PET [\%] & Dose [Gy] & SIB [Gy] & Single dose [Gy] & $\mathrm{TCP}_{\text {conv }}[\%]$ & TCP Increase [\%] \\
\hline 0.04 & 1.5 & 2.2 & 81 & 78 & 90 & 2 & 77.2 & 14.9 \\
\hline 0.04 & 1.5 & 2.2 & 64 & 78 & 90 & 2 & 77.2 & 11.5 \\
\hline 0.06 & 3.1 & 2.2 & 81 & 78 & 90 & 2 & 77.2 & 14.1 \\
\hline 0.06 & 3.1 & 2.2 & 64 & 78 & 90 & 2 & 77.2 & 11.0 \\
\hline 0.08 & 8.3 & 2.2 & 81 & 78 & 90 & 2 & 77.2 & 13.1 \\
\hline 0.08 & 8.3 & 2.2 & 64 & 78 & 90 & 2 & 77.2 & 10.1 \\
\hline
\end{tabular}

Calculation of the TCP-increase after boosting choline PET positive regions within the prostate up to 90 Gy, higher homogeneous whole prostate dose. $\alpha / \beta$ was analytically determined in order to achieve agreement between calculated TCD50 and TCD50 obtained by Cheung et al. $\gamma 50$ was again taken from Cheung's data (ASTRO definition).

Table 7 TCP-increase for different sensitivities of PET, low whole prostate dose, different $\alpha / \beta$ values, calculated $\alpha$ and low $\gamma 50$ (CN +2 definition)

\begin{tabular}{|c|c|c|c|c|c|c|c|c|}
\hline$\alpha\left[\mathrm{Gy}^{-1}\right]$ & $\alpha / \beta[G y]$ & $\gamma 50$ & Det. rate PET [\%] & Dose [Gy] & SIB [Gy] & Single dose [Gy] & $\mathrm{TCP}_{\text {conv }}[\%]$ & $\begin{array}{c}\text { TCP Increase } \\
{[\%]}\end{array}$ \\
\hline 0.03 & 1.5 & 1.4 & 81 & 74 & 90 & 2 & 80 & 13.2 \\
\hline 0.03 & 1.5 & 1.4 & 64 & 74 & 90 & 2 & 80 & 10.3 \\
\hline 0.04 & 3.1 & 1.4 & 81 & 74 & 90 & 2 & 80 & 12.6 \\
\hline 0.04 & 3.1 & 1.4 & 64 & 74 & 90 & 2 & 80 & 9.8 \\
\hline 0.06 & 8.3 & 1.4 & 81 & 74 & 90 & 2 & 80 & 11.6 \\
\hline 0.06 & 8.3 & 1.4 & 64 & 74 & 90 & 2 & 80 & 9.0 \\
\hline
\end{tabular}

Calculation of the TCP-increase after boosting choline PET positive regions within the prostate up to 90 Gy. $\alpha / \beta$ was set to either Fowler's/Wang's or Valdagni's value, $\alpha$ was analytically determined in order to achieve agreement between calculated TCD50 and TCD50 obtained by Cheung et al. $\gamma 50$ was again taken from Cheung's data ( $\mathrm{CN}+2$ definition). 
Table 8 TCP-increase for different sensitivities of PET, high whole prostate dose, different $\alpha / \beta$ values, calculated $\alpha$ and low $\gamma 50(\mathrm{CN}+2$ definition)

\begin{tabular}{|c|c|c|c|c|c|c|c|c|}
\hline$\alpha\left[\mathrm{Gy}^{-1}\right]$ & $\alpha / \beta[G y]$ & $\gamma 50$ & Det. rate PET [\%] & Dose [Gy] & SIB [Gy] & Single dose [Gy] & $\mathrm{TCP}_{\text {conv }}[\%]$ & $\begin{array}{c}\text { TCP Increase } \\
{[\%]}\end{array}$ \\
\hline 0.03 & 1.5 & 1.4 & 81 & 78 & 90 & 2 & 84.5 & 9.1 \\
\hline 0.03 & 1.5 & 1.4 & 64 & 78 & 90 & 2 & 84.5 & 7.1 \\
\hline 0.04 & 3.1 & 1.4 & 81 & 78 & 90 & 2 & 84.5 & 8.5 \\
\hline 0.04 & 3.1 & 1.4 & 64 & 78 & 90 & 2 & 84.5 & 6.6 \\
\hline 0.06 & 8.3 & 1.4 & 81 & 78 & 90 & 2 & 84.5 & 7.7 \\
\hline 0.06 & 8.3 & 1.4 & 64 & 78 & 90 & 2 & 84.5 & 6.0 \\
\hline
\end{tabular}

Calculation of the TCP-increase after boosting choline PET positive regions within the prostate up to $90 \mathrm{~Gy}$, low homogeneous dose $78 \mathrm{~Gy}$. $\alpha / \beta$ was set to either Fowler's/Wang's or Valdagni's result, $\alpha$ was analytically determined in order to achieve agreement between calculated TCD50 and TCD50 obtained by Cheung et al. $\gamma 50$ was again taken from Cheung's data $(\mathrm{CN}+2$ definition).

the definition of tumor control, the dose used for the treatment of the whole organ and the $\alpha / \beta$ values.

We observed a high variation between the outcomes based on different initial assumptions. A critical limitation is the fact that there is no chance to derive $\alpha / \beta$ and $\alpha$ values for the calculation of dose-response relationships from the trial by Cheung et al. (the best data available to date) since a single fixed fractionation schedule was applied [35].

In keeping with this several inconsistencies occurred (Table 5, Table 6, Table 7 and Table 8): the calculated $\alpha$ values did not fit their counterpart in literature except for Fowler's data where the deviation was small. In this case the dependence on $\gamma 50$ and the detection rate of choline PET became more important.

On the one hand, $\gamma 50$ depends on the failure definition and data are different with longer follow-up data, and at present the confidence interval is still wide as $\gamma 50$ $=2.2[1.1-3.2,95 \% \mathrm{CI}]$ (ASTRO definition) or $\gamma 50=1.4$ [0.2-2.5, 95\% CI] $(\mathrm{CN}+2$ definition $)$.

On the other hand, a study from Farsad et al. demonstrated that $\mathrm{C}$-11-choline PET/CT has a relatively high rate of false-negative results on a sextant basis. In addition it has been clearly shown that non-malignant prostatic disorders may induce an increased ${ }^{11} \mathrm{C}$-choline uptake [62]. Our model calculations are not dependent on specificity as the irradiation of non-infiltrated voxels does not influence TCP but this will lead to unessentially big SIB target volumes.

Taken together, the relatively high efficacy rates of an IMRT based SIB are potentially overestimating the real benefit (Table 5, Table 6, Table 7 and Table 8, between $7.1 \%$ and $22.2 \%$ ). Patient setup errors as well as intrafraction motion of the prostate were not considered throughout the whole estimation process which could potentially hamper the results in a negative way [63-67]. Another important factor influencing tumor control was neglected in the model: the risk of regional, i.e. pelvic nodal and/or systemic failure. This may be a potential source of limiting the effectiveness of this approach as it was assumed that local control entails biochemical control; in this regard a single cancer cell outside the prostate could violate this assumption and diminish tumor control.

Despite all of our considerations our model data are not in contrast to data provided by Kim et al. [68] claiming that selective boosting is more effective than homogeneous dose escalation as sparing of normal tissue is easier to achieve.

Furthermore, risk-adaptive optimization increases the therapeutic ratio as compared to conventional selective boosting IMRT. In another paper Kim et al. derive similar results, but mention the importance of the underlying imaging modality and consecutively their sensitivity in detecting occult tumor cells [69].

Utilizing an IMRT boost is an elegant technique but one has to mention another classical but suitable method: With brachytherapy the doses to the organs at risk are lower or similar to IMRT-only. Dose escalation for prostate tumors may also be easily achieved by brachytherapy alone [70].

\section{Conclusions}

Regarding treatment planning in radiotherapy, choline PET may offer some advantages in terms of staging, tumor delineation and the description of biological processes. However, a TCP-increase related to any IMRT SIB on choline PET positive regions has to be considered as realistically low.

Additional file 1: This file contains a sheet where parameters like choline PET sensitivity/specificity, $\alpha, \alpha / \beta, \gamma 50$, TCD50, dose, SIB dose and single dose can be specified and a sheet carrying out all necessary calculation steps.

Click here for file

[http://www.biomedcentral.com/content/supplementary/1748-717X-5-23S1.XLS ]

\section{Abbreviations}

RT: radiotherapy; IMRT: intensity-modulated radiotherapy; SIB: simultaneous integrated boost; PTV: Planning target volume; LQ: linear-quadratic; TCP: 
tumor control probability; TCD50: tumor control dose 50\%; PET: positron emission tomography; SUV: standardized uptake value; Cl: confidence interval; SF: surviving fraction; EBRT: external beam radiotherapy; PSA: prostate-specific antigen; ASTRO: American Society for Therapeutic Radiology and Oncology; CN: current nadir; FWHM: full width at half maximum.

\section{Author details}

${ }^{1}$ Department of Radiation Oncology, Ludwig-Maximilians-University München, Marchioninistr. 15, 81377 München, Germany. ${ }^{2}$ Department of Nuclear Medicine, Ludwig-Maximilians-University München, Marchioninistr. 15, 81377 München, Germany.

\section{Authors' contributions}

MN developed the underlying mathematical model and wrote the manuscript. PB participated in the preparation of the manuscript. UG and $C B$ provided the idea and participated in the conception as well as the preparation of the manuscript. All authors read and approved the final manuscript.

\section{Competing interests}

The authors declare that they have no competing interests.

Received: 19 January 2010 Accepted: 18 March 2010

Published: 18 March 2010

\section{References}

1. Pollack A, Zagars GK, Starkschall G, Antolak JA, Lee JJ, Huang E, von Eschenbach AC, Kuban DA, Rosen I: Prostate cancer radiation dose response: Results of the M. D. Anderson phase III randomized trial. International Journal of Radiation Oncology Biology Physics 2002, 53(5):1097-1105.

2. Zelefsky MJ, Leibel SA, Gaudin PB, Kutcher GJ, Fleshner NE, Venkatramen ES, Reuter VE, Fair WR, Ling CC, Fuks Z: Dose escalation with threedimensional conformal radiation therapy affects the outcome in prostate cancer. International Journal of Radiation Oncology Biology Physics 1998, 41(3):491-500.

3. Hanks GE, Hanlon AL, Epstein B, Horwitz EM: Dose response in prostate cancer with 8-12 years' follow-up. Elsevier Science Inc 2002, 427-435.

4. Bey $P$, Carrie C, Beckendorf V, Ginestet C, Aletti P, Madelis G, Luporsi E, Pommier P, Cowen D, Gonzague-Casabianca L, et al: Dose escalation with 3D-CRT in prostate cancer: French study of dose escalation with conformal 3 D radiotherapy in prostate cancer - Preliminary results. International Journal of Radiation Oncology Biology Physics 2000, 48(2):513-517.

5. Boersma LJ, Brink van den M, Bruce AM, Shouman T, Gras L, te Velde A, Lebesque JV: Estimation of the incidence of late bladder and rectum complications after high-dose (70-78 Gy) conformal radiotherapy for prostate cancer, using dose-volume histograms. International Journal of Radiation Oncology Biology Physics 1998, 41(1):83-92.

6. Forman JD, Duclos M, Shamsa F, Porter AT, Orton C: Hyperfractionated conformal radiotherapy in locally advanced prostate cancer: Results of a dose escalation study. International Journal of Radiation Oncology Biology Physics 1996, 34(3):655-662.

7. Welz S, Nyazi M, Belka C, Ganswindt U: Surgery vs. radiotherapy in localized prostate cancer. Which is best? Radiation Oncology 2008, 3.

8. Ganswindt U, Paulsen F, Anastasiadis AG, Stenzl A, Bamberg M, Belka C: 70 Gy or more: which dose for which prostate cancer? I Cancer Res Clin Oncol 2005, 131(7):407-419.

9. Tanderup K, Olsen DR, Grau C: Dose painting: Art or science? Radiotherapy and Oncology 2006, 79(3):245-248.

10. Al-Mamgani A, Heemsbergen WD, Peeters STH, Lebesque JV: ROLE OF INTENSITY-MODULATED RADIOTHERAPY IN REDUCING TOXICITY IN DOSE ESCALATION FOR LOCALIZED PROSTATE CANCER. International Journal of Radiation Oncology Biology Physics 2009, 73(3):685-691.

11. Pickett B, Vigneault E, Kurhanewicz J, Verhey L, Roach M: Static field intensity modulation to treat a dominant intra-prostatic lesion to $90 \mathrm{~Gy}$ compared to seven field 3-dimensional radiotherapy. Int I Radiat Oncol Biol Phys 1999, 44(4):921-929.

12. Xia P, Pickett B, Vigneault E, Verhey $\amalg$, Roach M: Forward or inversely planned segmental multileaf collimator IMRT and sequential tomotherapy to treat multiple dominant intraprostatic lesions of prostate cancer to 90 Gy. Int I Radiat Oncol Biol Phys 2001, 51(1):244-254.
13. Dogan N, Wu Y, Hagan MP: Simultaneous-integrated boost (SIB) IMRT for treatment of indermediate-risk prostate cancer with nodal irradiation. International Journal of Radiation Oncology Biology Physics 2006, 66(3):2805.

14. Singh AK, Guion P, Sears-Crouse N, Ullman K, Smith S, Albert PS, Fichtinger G, Choyke PL, Xu S, Kruecker J, et al: Simultaneous integrated boost of biopsy proven, MRI defined dominant intra-prostatic lesions to 95 Gray with IMRT: early results of a phase I NCl study. Radiation Oncology 2007, 2.

15. Li X, Wang JZ, Jursinic P, Lawton CA: IMRT simultaneous integrated boost for high-risk prostate cancer. International Journal of Radiation Oncology Biology Physics 2004, 60(1):2486

16. Pinkawa M, Piroth MD, Fischedick K, Nussen S, Klotz J, Holy R, Eble MJ: Selfassessed bowel toxicity after external beam radiotherapy for prostate cancer-predictive factors on irritative symptoms, incontinence and rectal bleeding. Radiat Oncol 2009, 4:36.

17. Onal C, Topkan E, Efe E, Yavuz M, Sonmez S, Yavuz A: Comparison of rectal volume definition techniques and their influence on rectal toxicity in patients with prostate cancer treated with $3 \mathrm{D}$ conformal radiotherapy: a dose-volume analysis. Radiat Oncol 2009, 4:14.

18. Martin JM, Bayley A, Bristow R, Chung P, Gospodarowicz M, Menard C, Milosevic M, Rosewall T, Warde PR, Catton CN: Image guided dose escalated prostate radiotherapy: still room to improve. Radiat Oncol 2009, 4:50.

19. Guckenberger M, Baier K, Richter A, Vordermark D, Flentje M: Does intensity modulated radiation therapy (IMRT) prevent additional toxicity of treating the pelvic lymph nodes compared to treatment of the prostate only? Radiat Oncol 2008, 3:3.

20. Ghadjar P, Vock J, Vetterli D, Manser P, Bigler R, Tille J, Madlung A, Behrensmeier F, Mini R, Aebersold DM: Acute and late toxicity in prostate cancer patients treated by dose escalated intensity modulated radiation therapy and organ tracking. Radiat Oncol 2008, 3:35.

21. Ghadjar P, Matzinger O, Isaak B, Behrensmeier F, Stroux A, Rentsch CA, Thalmann GN, Aebersold DM: Association of urethral toxicity with dose exposure in combined high-dose-rate brachytherapy and intensitymodulated radiation therapy in intermediate- and high-risk prostate cancer. Radiotherapy and Oncology 2009, 91(2):237-242.

22. Li XA, Wang JZ, Jursinic PA, Lawton CA, Wang D: Dosimetric advantages of IMRT simultaneous integrated boost for high-risk prostate cancer. International Journal of Radiation Oncology Biology Physics 2005, 61(4):1251-1257.

23. Fonteyne V, Villeirs G, Speleers B, De Neve W, De Wagter C, Lumen N, De Meerleer $\mathrm{G}$ : Intensity-modulated radiotherapy as primary therapy for prostate cancer: Report on acute toxicity after dose escalation with simultaneous integrated boost to intraprostatic lesion. International Journal of Radiation Oncology Biology Physics 2008, 72(3):799-807.

24. Weber DC, Wang H, Cozzi L, Dipasquale G, Khan HG, Ratib O, Rouzaud M, Vees H, Zaidi H, Miralbell R: RapidArc, intensity modulated photon and proton techniques for recurrent prostate cancer in previously irradiated patients: a treatment planning comparison study. Radiat Oncol 2009, 4:34.

25. Yuen J, Rodrigues G, Trenka K, Coad T, Yartsev S, D'Souza D, Lock M, Bauman G: Comparing two strategies of dynamic intensity modulated radiation therapy (dIMRT) with 3-dimensional conformal radiation therapy (3DCRT) in the hypofractionated treatment of high-risk prostate cancer. Radiat Oncol 2008, 3:1.

26. Groves AM, Win T, Ben Haim S, Ell PJ: Non-[F-18]FDG PET in clinical oncology. Lancet Oncology 2007, 8(9):822-830.

27. Picchio M, Crivellaro C, Giovacchini G, Gianolli L, Messa C: PET-CT for treatment planning in prostate cancer. Q I NuCl Med Mol Imag 2009, 53(2):245-268.

28. Reske SN, Blumstein NM, Glatting G: [C-11]choline PET/CT imaging in occult local relapse of prostate cancer after radical prostatectomy. European Journal of Nuclear Medicine and Molecular Imaging 2008, 35(1):9-17.

29. Grosu AL, Piert M, Weber WA, Jeremic B, Picchio M, Schratzenstaller U, Zimmermann FB, Schwaiger M, Molls M: Positron emission tomography for radiation treatment planning. Strahlentherapie Und Onkologie 2005, 181(8):483-499.

30. Ganswindt U, Paulsen F, Alber M, Bares R, Bamberg M, Belka C: Intensitymodulated radiotherapy (IMRT) for lymph node-positive patients in prostate cancer under consideration of C-11-choline-PET data - first clinical experiences. Strahlentherapie Und Onkologie 2006, 182:71-71. 
31. Ciernik IF, Brown DW, Schmid D, Hany T, Egli P, Davis JB: 3D-Segmentation of the F-18-choline PET signal for target volume definition in radiation therapy of the prostate. Technology in Cancer Research \& Treatment 2007, 6(1):23-30.

32. Stewart RD, Li XA: BGRT: Biologically guided radiation therapy - The future is fast approaching!. Medical Physics 2007, 34:3739-3751.

33. Payne GS, Leach MO: Applications of magnetic resonance spectroscopy in radiotherapy treatment planning. British Journal of Radiology 2006, 79: S16-S26.

34. van Lin E, Futterer JJ, Heumink S, Vight Van Der LP, Hoffmann AL, Van Kollenburg P, Huisman HJJ, Scheenen TWJ, Witjes JA, Leer JWK, et al: IMRT boost dose planning on dominant intraprostatic lesions: Gold markerbased three-dimensional fusion of $\mathrm{CT}$ with dynamic contrast-enhanced and $\mathrm{H}$-1-spectroscopic MRI. International Journal of Radiation Oncology Biology Physics 2006, 65(1):291-303.

35. Cheung R, Tucker SL, Lee AK, De Crevoisier R, Dong L, Kamat A, Pisters L, Kuban D: Dose-response characteristics of low- and intermediate-risk prostate cancer treated with external beam radiotherapy. Elsevier Science Inc 2005, 993-1002

36. Thames H, Kuban D, Levy L, Horwitz EM, Kupelian P, Martinez A, Michalski J, Pisansky T, Sandler H, Shipley W, et al: Comparison of alternative biochemical failure definitions' based on clinical outcome in 4839 prostate cancer patients treated by external beam radiotherapy between 1986 and 1995. International Journal of Radiation Oncology Biology Physics 2003, 57(4):929-943.

37. Kuban DA, Thames HD, Levy LB: Radiation for prostate cancer: use of biochemical failure as an endpoint following radiotherapy. World Journal of Urology 2003, 21(4):253-264.

38. Kuban DA, Thames HD, Levy LB, Horwitz EM, Kupelian PA, Martinez AA, Michalski JM, Pisansky TM, Sandler HM, Shipley WU, et al: Failure definitiondependent differences in outcome following radiation for localized prostate cancer. Can one size fit all? International Journal of Radiation Oncology Biology Physics 2003, 57(2 Supplement):S146-S147.

39. Munro TR, Gilbert CW: THE RELATION BETWEEN TUMOUR LETHAL DOSES AND THE RADIOSENSITIVITY OF TUMOUR CELLS. British Journal of Radiology 1961, 34(400):246-251.

40. O'Rourke SFC, MCAneney H, Hillen T: Linear quadratic and tumour control probability modelling in external beam radiotherapy. Journal of Mathematical Biology 2009, 58(4-5):799-817.

41. Garcia LM, Wilkins DE, Raaphorst GP: alpha/beta ratio: A dose range dependence study. International Journal of Radiation Oncology Biology Physics 2007, 67(2):587-593

42. Fowler JF, Ritter MA, Fenwick JD, Chappell RJ: How low is the alpha/beta ratio for prostate cancer? In regard to Wang et al., IJROBP 2003; 55: 194203. International Journal of Radiation Oncology Biology Physics 2003, 57(2):593-595

43. Fowler J, Chappell R, Ritter M: Is alpha/beta for prostate tumors really low? International Journal of Radiation Oncology Biology Physics 2001, 50(4):1021-1031

44. D'Souza WD, Thames HD: Is the alpha/beta ratio for prostate cancer low? International Journal of Radiation Oncology Biology Physics 2001, 51(1):1-3.

45. Chappell R, Fowler J, Ritter M: New data on the value of alpha/beta Evidence mounts that it is low. (vol 60, pg 2004). International Journal of Radiation Oncology Biology Physics 1002, 61(2):635-635.

46. Bentzen SM, Ritter MA: The alpha/beta ratio for prostate cancer: What is it, really? Radiotherapy and Oncology 2005, 76(1):1-3.

47. Kal HB, Van Gellekom MPR: How low is the alpha/beta ratio for prostate cancer? International Journal of Radiation Oncology Biology Physics 2003, 57(4):1116-1121

48. Valdagni R, Italia C, Montanaro P, Lanceni A, Lattuada P, Magnani T, Fiorino C, Nahum A: Is the alpha-beta ratio of prostate cancer really low? A prospective, non-randomized trial comparing standard and hyperfractionated conformal radiation therapy. Radiotherapy and Oncology 2005, 75(1):74-82.

49. Wang JZ, Guerrero M, Li XA: How low is the alpha/beta ratio for prostate cancer? International Journal of Radiation Oncology Biology Physics 2003, 55(1):194-203.

50. Wang JZ, Li XA, Yu CX, DiBiase SJ: The low alpha/beta ratio for prostate cancer: What does the clinical outcome of HDR brachytherapy tell. International Journal of Radiation Oncology Biology Physics 2003, 57(4):1101-1108.
51. Williams SG, Taylor JMG, Liu N, Tra Y, Duchesne GM, Kestin LL, Martinez A, Pratt GR, Sandler H: Use of individual fraction size data from 3756 patients to directly determine the alpha/beta ratio of prostate cancer. International Journal of Radiation Oncology Biology Physics 2007, 68(1):24-33.

52. Li XA, Wang JZ, Stewart RD, Dibiase SJ, Wang D, Lawton CA: Designing equivalent treatment regimens for prostate radiotherapy based on equivalent uniform dose. British Journal of Radiology 2008, 81(961):59-68.

53. Tome WA, Fowler JF: On cold spots in tumor subvolumes. Medical Physics 2002, 29(7):1590-1598.

54. Reske SN, Blumstein NM, Glatting G: Advancement of PET and PET/CT in prostate carcinoma. Urologe 2006, 45(6).

55. Krause BJ, Souvatzoglou M, Tuncel M, Herrmann K, Buck AK, Praus $C$, Schuster T, Geinitz H, Treiber U, Schwaiger M: The detection rate of [C-11] Choline-PET/CT depends on the serum PSA-value in patients with biochemical recurrence of prostate cancer. European Journal of Nuclear Medicine and Molecular Imaging 2008, 35(1):18-23.

56. Scattoni V, Picchio M, Suardi N, Messa C, Freschi M, Roscigno M, Da Pozzo L, Bocciardi A, Rigatti P, Fazio F: Detection of lymph-node metastases with integrated [C-11]choline PET/CT in patients with PSA failure after radical retropubic prostatectomy: Results confirmed by open pelvic-retroperitoneal lymphadenectomy. Eur Urol 2007, 52(2):423-429.

57. Soret M, Bacharach SL, Buvat I: Partial-volume effect in PET tumor imaging. J NuCl Med 2007, 48(6):932-945.

58. Ganswindt U, Stenzl A, Bamberg M, Belka C: Adjuvant radiotherapy for patients with locally advanced prostate cancer - A new standard? Eu Urol 2008, 54(3):528-542.

59. Ganswindt U, Belka C: Radiotherapy in prostate cancer. Urologe 2008 , 47(9):1245-1254

60. King CR, Kapp DS: Radiotherapy after prostatectomy: Is the evidence for dose escalation out there? International Journal of Radiation Oncology Biology Physics 2008, 71(2):346-350.

61. Warkentin B, Stavrev P, Stavreva NA, Fallone BG: Limitations of a TCP model incorporating population heterogeneity. Physics in Medicine and Biology 2005, 50(15):3571-3588.

62. Farsad M, Schiavina R, Castellucci P, Nanni C, Corti B, Martorana G, Canini R, Grigioni W, Boschi S, Marengo M, et al: Detection and localization of prostate cancer: Correlation of C-11-choline PET/CT with histopathologic step-section analysis. Journal of Nuclear Medicine 2005, 46(10):1642-1649.

63. Krengli M, Gaiano S, Mones E, Ballare A, Beldi D, Bolchini C, Loi G: Reproducibility of patient setup by surface image registration system in conformal radiotherapy of prostate cancer. Radiat Oncol 2009, 4:9.

64. Nyholm T, Nyberg M, Karlsson MG, Karlsson M: Systematisation of spatial uncertainties for comparison between a MR and a CT-based radiotherapy workflow for prostate treatments. Radiat Oncol 2009, 4:54.

65. Graf R, Wust P, Budach V, Boehmer D: Potentials of on-line repositioning based on implanted fiducial markers and electronic portal imaging in prostate cancer radiotherapy. Radiat Oncol 2009, 4:13.

66. Lips IM, Dehnad H, van Gils CH, Boeken Kruger AE, Heide van der UA, van Vulpen M: High-dose intensity-modulated radiotherapy for prostate cancer using daily fiducial marker-based position verification: acute and late toxicity in 331 patients. Radiat Oncol 2008, 3:15.

67. Boda-Heggemann J, Kohler FM, Wertz H, Ehmann M, Hermann B, Riesenacker N, Kupper B, Lohr F, Wenz F: Intrafraction motion of the prostate during an IMRT session: a fiducial-based 3D measurement with Cone-beam CT. Radiat Oncol 2008, 3:37.

68. Kim Y, Tome WA: Is it beneficial to selectively boost high-risk tumor subvolumes? A comparison of selectively boosting high-risk tumor subvolumes versus homogeneous dose escalation of the entire tumor based on equivalent EUD plans. Acta Oncologica 2008, 47(5):906-916.

69. Kim $Y$, Tome WA: On the impact of functional imaging accuracy on selective boosting IMRT. Physica Medica 2009, 25(1):12-24.

70. Pieters BR, Kamer van de JB, van Herten YR, van Wieringen N, D'Olieslager GM, Heide van der UA, Koning CC: Comparison of biologically equivalent dose-volume parameters for the treatment of prostate cancer with concomitant boost IMRT versus IMRT combined with brachytherapy. Radiother Oncol 2008, 88(1):46-52.

doi:10.1186/1748-717X-5-23

Cite this article as: Niyazi et al:: Choline PET based dose-painting in prostate cancer - Modelling of dose effects. Radiation Oncology 2010 5:23. 